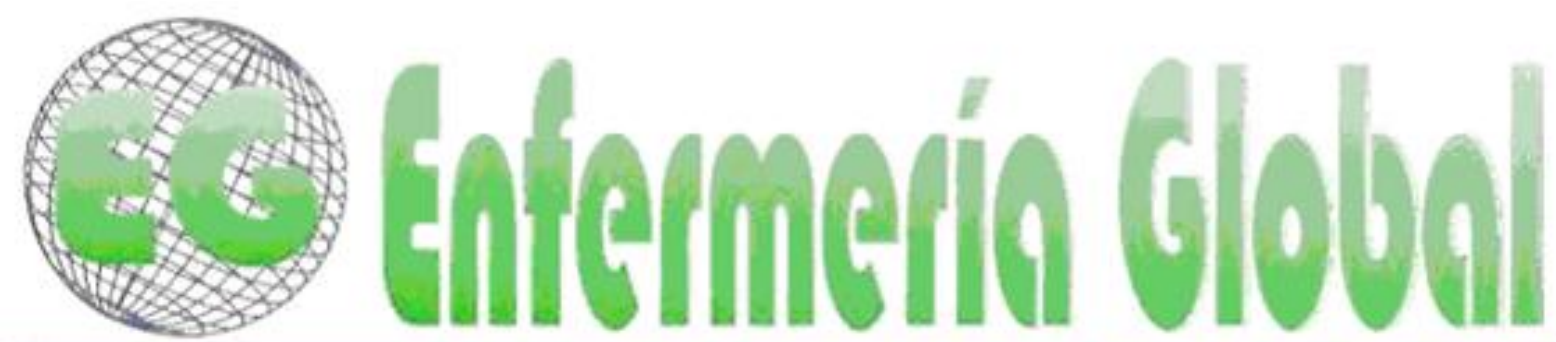

ISSN 1695-6141

$N^{\circ} 44$

Revista electrónica trimestral de Enfermería

www.um.es/egloball

Octubre 2016

DOCENCIA - INVESTIGACIÓN

\title{
Influencia de la actividad física y los hábitos nutricionales sobre el riesgo de síndrome metabólico
}

Influence of physical activity and nutritional habits on the risk of metabolic syndrome

\section{${ }^{*}$ Morales, $\mathrm{M}^{\mathrm{a}}$ Isabel Arandojo ${ }^{* *}$ Pacheco Delgado, Valeriano ${ }^{* * *}$ Morales Bonilla, José Antonio}

\begin{abstract}
*Enfermera. Doctoranda en la Facultad de Enfermería, Fisioterapia y Podología de la UCM. Servicio Médico del Colegio de Guardias Jóvenes "Duque de Ahumada" de la Guardia Civil. Valdemoro, Madrid. E-mail: maribelarandojo@gmail.com **Enfermero. Servicio Médico de la Escuela de Tráfico de la Guardia Civil. Mérida. Badajoz. **** Enfermero. Servicio Médico del Colegio de Guardias Jóvenes

“ Duque de Ahumada” de la Guardia Civil. Valdemoro, Madrid. España.
\end{abstract}

Palabras clave: Obesidad; sedentarismo; síndrome metabólico; enfermedad cardiovascular.

Keywords: Obesity; sedentary lifestyle; metabolic syndrome; cardiovascular disease.

\section{RESUMEN}

La conjunción de varios factores de riesgo originados por la obesidad, el sedentarismo o por diversos factores genéticos, lleva a padecer el llamado "Síndrome Metabólico", que incrementa la posibilidad de desarrollar enfermedades cardiovasculares.

Objetivos: Describir el riesgo de padecer enfermedades cardiovasculares que tienen los miembros de la Guardia Civil de un complejo militar de Madrid.

Material y método: Estudio descriptivo transversal, realizado mediante encuesta de 30 preguntas, analítica y medidas antropométricas al personal de la Guardia Civil que acudió al servicio médico de su acuartelamiento entre el 1 de septiembre y el 31 de octubre de 2014.

Resultados: Muestra de 76 sujetos de entre 20 y 62 años. El 7'89\% de los individuos tiene tres o más parámetros para el diagnóstico del Síndrome Metabólico.

Conclusiones: El sedentarismo y los malos hábitos nutricionales actúan negativamente en el perfil lipídico, TA y el IMC de los individuos, haciendo que estos tengan más probabilidades de padecer SM $y$, por tanto, enfermedades cardiovasculares. 


\section{ABSTRACT}

The combination of several risk factors caused by obesity, sedentary lifestyle or different genetic factors, leads to suffer "metabolic syndrome", which increases the chance of developing cardiovascular disease.

Objectives: Describe the risk of cardiovascular disease in members of Guardia Civil from a military complex in Madrid.

Material and method: Descriptive cross-sectional study carried out by a 30 questions-survey, analytics and anthropometric measurements to the Guardia Civil staff who came to the medical service of their quartering between September 1 and October 31, 2014.

Results: Sample of 76 subjects aged $20-62.7 ' 89 \%$ of individuals have three or more parameters related with metabolic syndrome diagnosis.

Conclusions: Sedentary lifestyle and poor nutritional habits act negatively in lipid profile, blood pressure and BMI, increasing the risk of metabolic syndrome and, therefore, cardiovascular diseases

\section{INTRODUCCIÓN}

En los últimos 50 años se han producido importantes cambios en nuestra sociedad. El hombre de hoy es más instruido, los adelantos científico-técnicos llegan a todos los rincones del planeta y se ha elevado el conocimiento humano en todos los sentidos, por lo que el trabajo es más intelectual y requiere un menor esfuerzo físico en general. Esto ha producido cambios en las condiciones sociales de vida y en la actividad de las personas, lo que ha conducido a que la población presente unos índices de exceso de peso cada vez más elevados y, con ello, aparezcan patologías asociadas que constituyen un grave problema de futuro para la salud pública ${ }^{1}$.

La salud es un valor social, que tiene relación con la calidad de la existencia humana. $Y$ es esta calidad y forma de vida de nuestra sociedad la que ha conducido a que la población presente unos índices de exceso de peso cada vez más elevados y con ello aparecen las patologías asociadas que constituyen un reto y un grave problema de futuro para la salud pública. Incluso algunos autores presentan la hipótesis de que la población infantil y adolescente actual pudiera ser la primera generación en ver reducida su esperanza de vida por esta causa, al aparecer ciertas enfermedades a edades más tempranas que en generaciones anteriores ${ }^{1}$.

El porcentaje de personas con sobrepeso está aumentando a una velocidad alarmante en todos los países industrializados. Se pasa más tiempo frente al televisor, el ordenador, la videoconsola o simplemente sentados, y menos tiempo haciendo ejercicio. El problema es de tal magnitud, que la Organización Mundial de la Salud (OMS) aporta los siguientes datos sobre la obesidad y el sobrepeso ${ }^{2}$ :

$>$ Desde 1980, la obesidad se ha más que doblado en todo el mundo.

> En 2008, 1400 millones de adultos (de 20 y más años) tenían sobrepeso. Dentro de este grupo, más de 200 millones de hombres y cerca de 300 millones de mujeres eran obesos.

$>$ El $65 \%$ de la población mundial vive en países donde el sobrepeso y la obesidad se cobran más vidas de personas que la insuficiencia ponderal (Indice de Masa Corporal < 18.5).

$>$ En 2010, alrededor de 40 millones de niños menores de cinco años tenían sobrepeso. 
En la misma nota informativa, la OMS establece como causa fundamental del sobrepeso y la obesidad el aumento en la ingesta de alimentos hipercalóricos y el descenso en la actividad física por una forma de vida cada vez más sedentaria. El peso corporal está, por tanto, en función del balance energético: Un balance energético positivo da lugar a una ganancia de peso, mientras que un balance energético negativo tiene el efecto contrario.

Es indiscutible que para vivir necesitamos comer y alimentarnos, pero alimentarse no es lo mismo que nutrirse. La alimentación son las acciones que permiten introducir en el organismo los alimentos que precisa. Nutrición, en cambio, es el conjunto de procesos mediante los cuales el cuerpo humano incorpora, transforma y utiliza los nutrientes suministrados con los alimentos ${ }^{3}$. Ocurre involuntariamente tras la ingestión de alimentos, por lo que depende de la calidad de la alimentación, que es muy importante que sea sana, suficiente, completa, armónica y adecuada. Se considera suficiente la alimentación que proporciona las cantidades óptimas de energía, los nutrientes esenciales para la vida y la cantidad de fibra dietética necesaria para una correcta función intestinal ${ }^{3}$.

Las necesidades de energía y nutrientes varían para cada individuo y dependen de su metabolismo basal (gasto de energía de un individuo en completo reposo físico y mental, 14 horas después de haber comido, en una habitación con temperatura de $20^{\circ} \mathrm{C}$ ), de su actividad diaria (ligera, moderada o pesada) y de su sexo, y puede variar desde $1450 \mathrm{kcal} /$ día para mujeres pequeñas sedentarias, hasta $4250 \mathrm{kcal} /$ día para hombres grandes muy activos ${ }^{2}$. Una dieta ideal aporta una cantidad suficiente de calorías para cubrir las necesidades energéticas, aunque no en exceso, ya que ello provocaría un aumento de peso.

Por otro lado, se puede definir el sedentarismo como la acción contraria a la realización de actividades que implican el movimiento corporal y que suponen un gasto energético superior al que correspondería al metabolismo basal ${ }^{4}$. Una persona sedentaria es la que invierte menos de un $10 \%$ de su gasto energético diario en la realización de actividades físicas que requieran al menos $4 \mathrm{MET}$. Un MET es el consumo energético de un individuo en estado de reposo (gasto metabólico basal), lo que equivale aproximadamente a $1 \mathrm{kcal}$ por kilo de peso y hora ${ }^{5}$. Se puede medir la duración, frecuencia, intensidad o entorno en el que se realiza la actividad para medir los METs, según el modelo de Ainsworth ${ }^{6}$, que adjudica un $n^{\circ}$ de METs a cada actividad. La intensidad o esfuerzo requerido depende de lo ejercitado que esté cada uno y de su forma física y se puede dividir en tres niveles de intensidad de acuerdo a los METs necesarios para realizarla ${ }^{6,7}$.

Actividad ligera: Menos de 3 METs, en actividades como pasear, ejercicios de elasticidad o sobre el suelo...

> Actividad moderada: De 3 a 6 METs, en actividades como caminar a paso rápido, tareas domésticas, jardinerías, levantar pesas, bailar...

> Actividad intensa: Más de 6 METs en actividades como correr, futbol y otros deportes competitivos, ejercicios aeróbicos...

La disminución progresiva de la actividad física en la vida social también ha hecho que varíe el proceso salud-enfermedad, porque es obvio que enfermedades como el infarto, el alcoholismo o los accidentes están más relacionados con las condiciones de vida de los individuos que con factores genéticos y fisiológicos ${ }^{8}$, convirtiéndose el 
sedentarismo en un factor de riesgo independiente y modificable para la enfermedad cardiovascular (ECV).

Puede definirse la obesidad como la acumulación de grasa en los tejidos, fenómeno que suele ir acompañado de un aumento del peso corporal. Este peso corporal está influido por la estatura y por ello, para evaluar el estado nutricional de un individuo, es necesario tenerlo en cuenta, por lo que a partir de ambas medidas se calcula el Indice de Masa Corporal $(\mathrm{IMC})^{9}$, que se interpreta como una medida de la forma en que la masa del individuo se distribuye por unidad de área corporal. Los parámetros que utiliza son simples y fáciles de obtener y su cálculo también es simple, mediante la fórmula "peso en $\mathrm{kg} /($ talla en metros)", por lo que se convierte en una herramienta muy útil. El resultado obtenido se compara dentro de unos valores, que se presentan en la Tabla I ${ }^{10}$ : Está altamente relacionado con la grasa corporal, por lo que es un método muy utilizado para describir la presencia de obesidad y clasificar al individuo según su grado de adiposidad, pero también es un buen descriptor de estados deficitarios. EI IMC solo no puede predecir el riesgo para la salud, pero la mayoría de los expertos coincide en que un índice superior a 30 (obesidad) no es saludable ${ }^{11}$. El hecho ser obeso sobrecarga al corazón y puede llevar a que se presenten graves problemas de salud, como cardiopatías, hipertensión arterial, diabetes tipo 2, etc., lo que convierte a la obesidad, junto con el sedentarismo, en otro factor de riesgo independiente y modificable para ECV.

Tabla I. Valores del IMC

\begin{tabular}{|lc|c|}
\hline \multicolumn{1}{|c}{ Clase } & $\begin{array}{c}\text { IMC }\left(\mathbf{k g} / \mathbf{m}^{2}\right) \\
<18,5\end{array}$ & $\begin{array}{c}\text { Riesgo } \\
\text { Bajo }\end{array}$ \\
\hline Peso insuficiente & $18,5-24,9$ & Medio \\
\hline Normal & $25,0-29,9$ & Moderado \\
\hline Sobrepeso & $30,0-34,9$ & Importante \\
\hline ObesidadI & $35,0-39,9$ & Grave \\
\hline ObesidadII & $>40,0$ & Muy grave \\
\hline ObesidadIII (Mórbida) & \multicolumn{2}{|c}{} \\
\hline
\end{tabular}

La ECV comprende un grupo de trastornos relacionados con la formación y desarrollo de procesos ateroscleróticos ${ }^{12}$. Las variables que aumentan las probabilidades de que una persona padezca una ECV se denominan factores de riesgo, que son múltiples, y la mayoría son modificables y asociados con los estilos de vida. Algunos de estos factores de riesgo cardiovascular se presentan juntos en ciertas personas y a esta conjunción de factores se le denomina "Síndrome Metabólico" (SM), también llamado

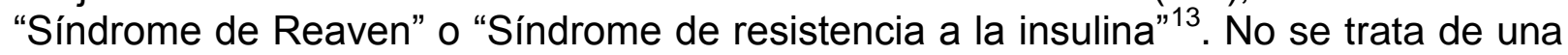
enfermedad única, sino de la asociación de problemas de salud que pueden aparecer de forma simultánea o secuencial en un mismo individuo ${ }^{14}$ y cuyos principales elementos son ${ }^{15}$ :

- Resistencia a la insulina: Aumento de la insulina basal para mantener la glucemia en un rango normal.

> Intolerancia a la glucosa: Los defectos de la acción de la insulina provocan la incapacidad de la hormona para suprimir la producción de glucosa por el hígado y riñón, además de alteraciones en el metabolismo de la glucosa.

> Obesidad abdominal, asociada al acúmulo de tejido adiposo en las vísceras abdominales. 
Dislipemia aterogénica: Aumento de la síntesis de Triglicéridos (TG) y disminución del colesterol HDL.

> Hipertensión arterial: Según la OMS, para que un paciente sea considerado hipertenso su presión arterial debe ser igual o superior a $140-90 \mathrm{mmHg}$ y según la NCEP-ATP III (National Cholesterol Education Program Adult Treatment Panel III) igual o superior a 135-85 $\mathrm{mmHg}$. Si un paciente tiene una presión arterial menor a esta cifra pero con tratamiento antihipertensivo, también será considerado hipertenso.

Existen varios criterios para el diagnóstico del SM propuestos por organismos internacionales de expertos, que difieren entre sí en sus componentes y sus puntos de corte ${ }^{14,16}$. (Tabla II). No existe una definición de SM consensuada internacionalmente, pero el criterio más utilizado para estudios poblacionales es el del NCEP-ATP $\mathrm{III}^{13}$, que considera que una persona tiene SM cuando tiene al menos tres de los problemas descritos anteriormente, por lo que será el criterio a seguir para el presente estudio.

Tabla II. Criterios diagnósticos para el Síndrome Metabólico

\begin{tabular}{|c|c|c|c|c|c|c|}
\hline $\begin{array}{c}\text { Criterios } \\
\text { diagnósticos }\end{array}$ & $\begin{array}{l}\text { OMS }^{1} \\
\text { (1999) }\end{array}$ & $\begin{array}{l}\text { EGIR }^{2} \\
(2002)\end{array}$ & $\begin{array}{c}\text { NCEP_ATP } \\
\text { IIP } \\
(2005)\end{array}$ & $\begin{array}{l}\mathrm{AACE}^{4} \\
(2003)\end{array}$ & $\begin{array}{l}\text { IDF }^{5} \\
(2005)\end{array}$ & $\begin{array}{l}\text { AHA/NHLBI } \\
(2005)\end{array}$ \\
\hline \begin{tabular}{|l|} 
Condiciones \\
\end{tabular} & $\begin{array}{c}\text { Aumento de } \\
\text { glucemia } \\
\text { basal, } \\
\text { Intolerancia a } \\
\text { la glucosa y/o } \\
\text { resistencia a la } \\
\text { insulina } \\
+ \\
\text { Dos o más de } \\
\text { los demás } \\
\text { criterios }\end{array}$ & \begin{tabular}{|c|} 
Resistencia a la \\
Insulina con \\
insulinemia $>25 \%$ \\
de los valores en \\
ayunas en no \\
diabéticos \\
+ \\
Dos o más de los \\
siguientes \\
criterios
\end{tabular} & $\begin{array}{c}\text { Presencia de } \\
\text { al menos } 3 \text { de } \\
\text { los siguientes } \\
\text { criterios }\end{array}$ & \begin{tabular}{|} 
Según juicio \\
clínico
\end{tabular} & \begin{tabular}{|c|} 
Perímetro \\
abdominal \\
elevado, \\
según las \\
etnias (da \\
valores para \\
europeos) \\
+ \\
Dos o más \\
de los de más \\
criterios
\end{tabular} & $\begin{array}{c}\text { Presencia de al } \\
\text { menos } 3 \text { de los } \\
\text { siguientes } \\
\text { criterios }\end{array}$ \\
\hline \begin{tabular}{|c|} 
Glucemia \\
basal (mg/dl)
\end{tabular} & $>110$ & $=110$ & $\begin{array}{c}=100 \\
\text { o tratamiento }\end{array}$ & $110-126$ & $\begin{array}{c}=100 \\
\text { o diabetes }\end{array}$ & $\begin{array}{c}=100 \\
\text { o tratamiento }\end{array}$ \\
\hline \begin{tabular}{|c|} 
Triglicéridos \\
(mg/dl)
\end{tabular} & $=150$ & $\begin{array}{c}=180 \\
\text { o tratamiento }\end{array}$ & $\begin{array}{c}=150 \\
\text { o tratamiento }\end{array}$ & $=150$ & $\begin{array}{c}=150 \\
\text { o tratamiento }\end{array}$ & $\begin{array}{c}=150 \\
\text { o tratamiento }\end{array}$ \\
\hline HDL (mg/dl) & $\begin{array}{c}\text { Hombres }<35 \\
\text { Mujeres }<39\end{array}$ & $\begin{array}{c}<40 \\
\text { o tratamiento }\end{array}$ & \begin{tabular}{|c|} 
Hombres $<40$ \\
Mujeres $<50$ \\
o tratamiento \\
\end{tabular} & $\begin{array}{l}\text { Hombres }<40 \\
\text { Mujeres }<50\end{array}$ & \begin{tabular}{|l|} 
Hombres $<40$ \\
Mujeres $<50$ \\
o tratamiento
\end{tabular} & $\begin{array}{c}\text { Hombres }<40 \\
\text { Mujeres }<50 \\
\text { o tratamiento }\end{array}$ \\
\hline $\begin{array}{c}\text { Presión } \\
\text { arterial } \\
(\mathrm{mmHg})\end{array}$ & $=140 / 90$ & $\begin{array}{c}=140 / 90 \\
\text { o tratamiento }\end{array}$ & $\begin{array}{c}=130 / 85 \\
\text { o tratamiento }\end{array}$ & $=130 / 85$ & $\begin{array}{c}=130 / 85 \\
\text { o tratamiento }\end{array}$ & $\begin{array}{l}\quad=130 / 85 \\
\text { o tratamiento }\end{array}$ \\
\hline $\begin{array}{l}\text { Perímetro } \\
\text { abdominal } \\
\text { (cm) }\end{array}$ & $\begin{array}{l}\text { Hombres }>90 \\
\text { Muje res }>85\end{array}$ & $\begin{array}{c}\text { Hombres }=90 \\
\text { Mujeres }=80\end{array}$ & $\mid \begin{array}{c}\text { Hombres }>102 \\
\text { Mujeres }>88\end{array}$ & $\begin{array}{c}\text { No lo } \\
\text { considera }\end{array}$ & $\left|\begin{array}{l}\text { Hombres }=94 \\
\text { Mujeres }=80 *\end{array}\right|$ & $\begin{array}{c}\text { Hombres }>102 \\
\text { Mujeres }>88\end{array}$ \\
\hline IMC $\left(\mathrm{kg} / \mathrm{m}^{2}\right)$ & $>30$ & No lo considera & $\begin{array}{c}\text { No lo } \\
\text { considera }\end{array}$ & $=25$ & $\begin{array}{c}\text { No lo } \\
\text { considera }\end{array}$ & No lo considera \\
\hline \multicolumn{7}{|c|}{$\begin{array}{l}\text { 1. Organización Mundial de la Salud } \\
\text { 2. Grupo Europeo para el Estudio de la Resistencia a la Insulina } \\
\text { 3. Panel de Expertos en Diagnóstico, Evaluación y Tratamiento de la Hipercolesterolemia en Adultos } \\
\text { 4. Colegio Americano de Endocrinología } \\
\text { 5. Federación Internacional de Diabetes } \\
\text { 6. Asociación Americana del Corazón/lnstituto Nacional del Corazón, Pulmón y Sangre }\end{array}$} \\
\hline
\end{tabular}

En definitiva, el SM es el nombre que se le da a un grupo de factores de riesgo que incrementan la posibilidad de desarrollar enfermedad cardiaca y otros problemas de salud como diabetes y accidente cerebro vascular ${ }^{17}$. Es considerado hoy día un problema clínico y de Salud Pública. Se estima que 1/4 de la población mundial lo tiene, lo que les ocasiona dos veces más probabilidad de morir y tres más de tener un infarto agudo de miocardio o un accidente cerebrovascular y cinco más de desarrollar diabetes mellitus tipo 2, además de aumentar la frecuencia de endurecimiento arterial, hipertrofia ventricular izquierda y disfunción diastólica, independientemente de presentar hipertensión arterial ${ }^{18}$. Los pacientes no sienten ningún síntoma, pero hay signos que pueden dar luz sobre su diagnóstico como una conjunción de obesidad 
abdominal, intolerancia a la glucosa o diabetes tipo 2, niveles elevados de LDL y TG en sangre, niveles bajos de HDL y presión arterial alta ${ }^{13}$.

La patogenia de este síndrome tiene muchos orígenes, pero la obesidad y un estilo de vida sedentario, junto con una dieta inadecuada y factores genéticos no bien conocidos, favorecen su aparición y sus crecientes incidencia y prevalencia ${ }^{19}$. En realidad, se trata de diferentes causas que actúan juntas. Algunas pueden ser controladas y otras no y las probabilidades de desarrollar un SM se incrementan con la edad.

\section{OBJETIVOS}

\section{General}

Describir el riesgo de padecer ECV, mediante el diagnóstico del SM, que tienen los miembros de la Guardia Civil destinados en un acuartelamiento de Madrid.

\section{Específicos}

1. Describir la estructura del grupo en cuanto a sus hábitos de alimentación y de realización de ejercicio físico.

2. Describir la prevalencia del SM en el grupo estudiado, así como los valores medios de las variables implicadas.

\section{MATERIAL Y MÉTODO}

Estudio descriptivo transversal realizado con miembros de la Guardia Civil destinados en Madrid, en un acuartelamiento donde se encuentran diversas unidades operativas y no operativas.

El estudio se ha realizado entre los individuos que acudieron al Servicio Médico entre el 1 de septiembre y el 31 de octubre de 2014 con el fin de realizarse una analítica, cada uno por sus motivos particulares. No se han considerado criterios de exclusión, siendo el único criterio de inclusión su voluntariedad de participar en el estudio. Todos los participantes accedieron a ofrecer su analítica para obtener los datos necesarios (glucosa, colesterol, HDL, LDL y triglicéridos) y a cumplimentar una encuesta de 30 preguntas sobre hábitos alimenticios y de actividad física, previamente validada y sometida a un estudio piloto. Se les midió la tensión arterial (TA), peso, altura y perímetro abdominal. Accedieron a participar todos los individuos a los que se preguntó, pero posteriormente se rechazó a 7 por errores en la cumplimentación de la encuesta. Al final, se consiguió una muestra de 76 individuos, 58 hombres y 18 mujeres, de entre 20 y 62 años.

Para el diagnóstico del SM se ha utilizado los criterios del NCEP-ATP III. Las muestras analíticas se obtuvieron después de 8-10 horas de ayuno y fueron procesadas todas en el mismo laboratorio, donde tienen como valores normales de referencia en mg/dl: glucosa 75-115, colesterol 50-220, HDL 35-120, LDL 50-130 y triglicéridos $45-150$.

El peso y la talla se han medido en una balanza calibrada con tallímetro, el IMC se calcula con la fórmula peso en $\mathrm{kg} /$ (talla en metros) $^{2}$, la circunferencia de la cintura se 
midió a la altura del ombligo, de pie y el con el abdomen descubierto y para la TA se ha utilizado un tensiómetro digital.

En cuanto a la actividad física realizada, se han obtenido datos sobre su tipo y frecuencia, duración y entorno en el que se realiza y se han utilizado los equivalentes metabólicos o METs para medir su intensidad, tanto si se trata de actividad física o actividad sedentaria.

Los datos se han procesado mediante los programas Excel 2010 y SPSS 20. Las variables cuantitativas se presentan como media \pm desviación estándar y como estadísticos se han utilizado la t de student para comparar medias y el coeficiente de correlación de Pearson ( $r$ ) para relacionar variables, ambas con un nivel de significación del $\leq 5 \%$ ( $p$-valor $\leq 0$ '05). Debido a la naturaleza descriptiva del estudio, no se han utilizado otros estadísticos para inferir resultados.

\section{RESULTADOS}

La muestra obtenida se compone de un 76 '32\% hombres y 23 '68\% mujeres. Esta desproporción entre sexos se debe al medio en el que se ha realizado el estudio, un acuartelamiento militar donde predomina el género masculino. La edad media es de 45 '34 \pm 10'66 años en los hombres y 33'00 \pm 11'52 años de las mujeres.

\section{Antropometría y datos analíticos}

Los hombres tienen valores significativamente más altos que las mujeres en peso, talla, IMC y perímetro abdominal, pero también en LDL y triglicéridos y las mujeres que tienen alguna alteración, es en los valores de glucosa y TA.

Sólo en los varones, se observa un considerable aumento en su perfil lipídico. El $6571 \%$ tiene elevados sus valores de LDL, el 31 '03\% el colesterol y el 10 '34\% los triglicéridos y un 20 '69\% tiene la TA por encima de $135 / 85 \mathrm{mmHg}$.

\section{IMC y dieta}

El IMC medio es de 27 '46 \pm 2'48 en hombres y 21'44 \pm 2'11 en mujeres y se relaciona con la edad ( $r=0$ '402, $p=0$ '012), de manera que en el intervalo de 50 a 59 años hay un 26 '31\% de personas con sobrepeso, varones todos ellos. El 52 ' $63 \%$ del total tiene sobrepeso, que sumado al $77^{\prime} 89 \%$ de obesos, nos aporta la información de que un total del 60 '52\% de los sujetos tienen problemas con su peso, aunque el $71^{\prime} 05 \%$ considera que lleva una dieta adecuada.

Se han distribuido los alimentos consumidos en dos grupos: saludables (recomendación de consumo diario) ${ }^{20}$ y no saludables (recomendación de consumo ocasional) encontrando que se consumen en más cantidad de los considerados saludables y menos los de consumo ocasional (Gráfico 1). Comparada la frecuencia de consumo en días a la semana con el IMC de los individuos, a medida que el IMC se incrementa, el consumo de alimentos saludables disminuye y los sujetos que tienen 3 o 4 parámetros para el diagnóstico de SM aumentan considerablemente el consumo de alimentos no saludables. 


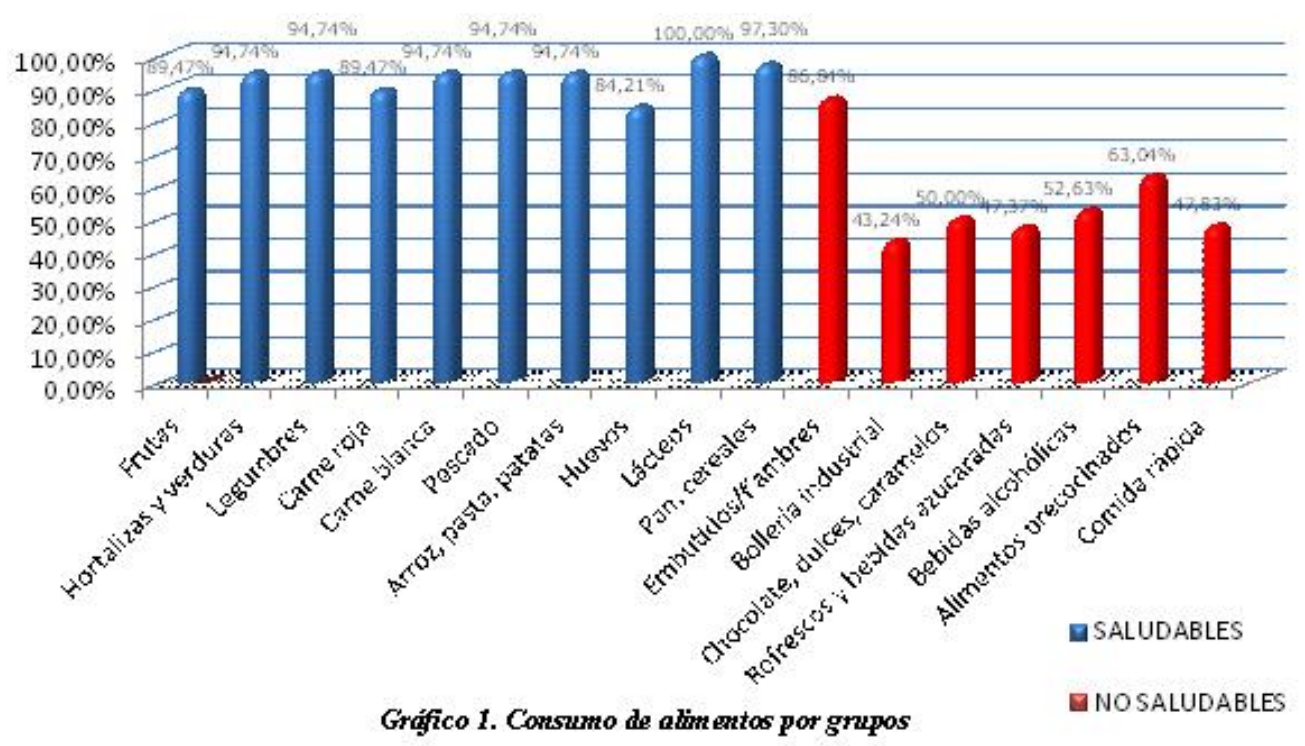

\section{Síndrome metabólico}

El 5'26\% de los individuos tiene 3 parámetros para el diagnóstico del SM y un 2'63\% tiene 4 parámetros, lo que hace que un $7{ }^{\prime} 89 \%$ de los sujetos tengan un mayor riesgo cardiovascular, todos ellos hombres. El $311^{\prime} 58 \%$ de los participantes tienen dos parámetros, por lo que estas personas no están diagnosticadas de SM pero tienen elevados la glucosa, el perímetro abdominal, o la tensión arterial.

El riesgo de SM también se ha relacionado con los alimentos que se consumen, según la distribución de estos en saludables y no saludables y se puede apreciar que, al igual que pasaba con el IMC, si bien los alimentos saludables se consumen más veces a la semana y con una frecuencia más o menos constante en todos los individuos, los sujetos que tienen 3 o 4 parámetros aumentan considerablemente el consumo de alimentos no saludables.

Los individuos que tienen hasta 29 años de edad tan sólo tienen un parámetro para el diagnóstico del SM 0 no tienen ninguno, pero el $n^{\circ}$ de factores de riesgo va aumentando con la edad, $\left(r=0^{\prime} 403, p=0,000\right)$, de manera que en el rango de 50 a 59 años, los individuos tienen entre 0 y 3 parámetros y los únicos que presentan 4 parámetros están en el rango de edad de 40-49 años.

\section{Ejercicio físico}

El $44^{\prime} 74 \%$ va a trabajar en un vehículo motorizado y el $28^{\prime} 95 \%$ lo hace andando. El $47^{\prime} 37 \%$ se echa la siesta y el $84{ }^{\prime} 21 \%$ dice hacer ejercicio físico para estar en forma $\left(33^{\prime} 33 \%\right)$ o porque les gusta $\left(31{ }^{\prime} 75 \%\right.$ ) mayoritariamente. Pero una media del $72{ }^{\prime} 81 \%$ no hace nunca o casi nunca ninguna de las actividades propuestas en la encuesta. La no actividad se mantiene por lo general en porcentajes más altos que la actividad. Parte de su tiempo libre lo utilizan para ver la televisión (100\%) o conectarse a Internet $\left(97^{\prime} 37 \%\right.$ ) y el $73^{\prime} 68 \%$ para leer. El $81^{\prime} 58 \%$ trabaja con el ordenador.

Aunque hay un aumento de actividad física moderada medida en METs en los individuos con dos parámetros, tanto la actividad ligera como intensa van disminuyendo a medida que los individuos tienen más parámetros de riesgo cardiovascular (Gráfico 2). 


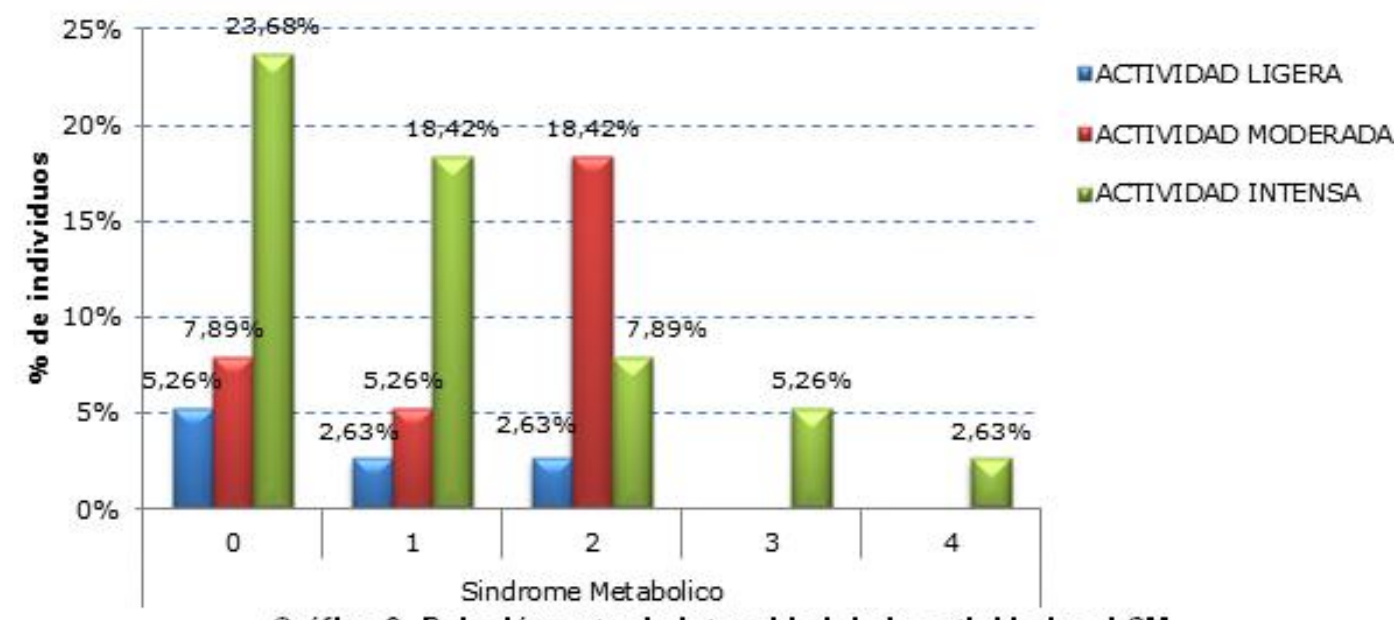

Gráfico 2. Relación entre la Intensidad de la actividad y el SM

\section{DISCUSIÓN}

Se ha analizado una muestra piloto de 76 individuos, que nos puede servir para describir cómo está la situación del SM en el grupo estudiado y plantearnos la posibilidad de profundizar más en un trabajo posterior. Pero en una valoración global, los resultados demuestran que la población estudiada presenta muchos de los factores relacionados con el SM y, por tanto, tienen un mayor riesgo de padecer ECV. Según uno de los últimos estudios realizados en nuestro país sobre población laboral española ${ }^{21}$, la prevalencia del SM es del $14{ }^{\prime} 4 \%$ en hombres y del $5^{\prime} 5 \%$ en mujeres. En nuestro estudio se demuestra que un $7,89 \%$ de los individuos que han participado tienen SM y no se ha obtenido resultado positivo de ninguna mujer, aunque en estas diferencias tienen mucho que ver las limitaciones de la muestra en cuanto a tamaño y distribución por sexos. Los casos encontrados con SM se diferencian de los que no lo tienen sobre todo por incremento en la TA Sistólica y los niveles de colesterol, aunque la prevalencia de hipertensión arterial y de hipercolesterolemia ha sido inferior a la de otros estudios ${ }^{22}$.

Es importante llamar la atención sobre la situación en la que se encuentran los individuos que, sin ser oficialmente diagnosticados de SM, también presentan factores de riesgo cardiovascular. Son los que tienen dos parámetros elevados, el $31{ }^{\prime} 58 \%$ de los participantes. Sólo les basta una pequeña variación en algún parámetro más para que aumente su riesgo de tener ECV. Es en este grupo de individuos donde habrá una mayor necesidad de prevención y educación sanitaria.

El perfil lipídico de los participantes está evidentemente elevado, sobre todo, en cuanto a los valores de LDL se refiere, sólo en los varones, y sus niveles son muy parecidos a los de otros estudios ${ }^{23}$. También los niveles de colesterol son considerables. Este es un dato para tener en cuenta ya que, aunque el LDL no se utiliza como parámetro para medir el SM, los individuos que tengan elevado el riesgo cardiovascular a causa de este síndrome verán aumentado mucho más su riesgo por la capacidad aterogénica del $\operatorname{LDL}^{13,24}$.

En cuanto a su alimentación, el 71 '05\% dice que su dieta es adecuada y existe con diferencia un mayor consumo de alimentos saludables que de los no saludables. Además, sólo un tercio de los individuos reconoce tener sobrepeso (31'58\%), mientras que el resto considera que su peso es normal. Sin embargo, la realidad de 
los datos recogidos nos confirma que estas proporciones prácticamente se intercambian, pues es un 36 ' $84 \%$ el que está dentro de su peso normal y un 52 '63\% tienen sobrepeso. Nadie reconoce en la encuesta tener obesidad, pero hay un 7'89\% de obesos. Si comparamos estos datos con los de la Encuesta Nacional de Salud 2011-12 (ENSE) ${ }^{25}$, la prevalencia de personas con sobrepeso y obesidad $(63$ ' $2 \%$ ) es prácticamente igual que el de este estudio (60'52\%). Por lo tanto, el mayor consumo que aparentemente existe de alimentos saludables con respecto a los menos recomendados no se refleja en el peso, sobre todo en los hombres, pues estos tienen un IMC medio de 27'46. Hay que tener en cuenta que, en la estimación del consumo de alimentos, el encuestado puede no acordarse de lo que comió y en algunos casos puede, de forma inconsciente, omitir el consumo de ciertos alimentos (como los dulces) o pensar que toma más de otros de lo que en realidad lo hace (como los lácteos). Este es un tipo de error, voluntario o involuntario, por parte de la persona encuestada, que puede responder lo que para él es socialmente aceptable, o en algún caso mentir ${ }^{26}$. Además, comen generalmente deprisa $(65,79 \%$ en menos de 30 minutos) y muchos no hacen las cinco comidas recomendadas ${ }^{17}$, lo que indica que sus hábitos alimenticios no son los más adecuados.

Sean cuales fueren la causas, la realidad indica que la ingesta de alimentos saludables disminuye cuanto mayor es el peso y, cuantos más parámetros tienen a favor del SM, más aumenta la frecuencia de consumo de alimentos poco recomendables, lo que contribuye al incremento de su peso y elevación de sus valores analíticos. El desconocimiento, por tanto, de lo que debe ser una buena alimentación, es quizá lo que hace considerar al 71 '05\% de los individuos que su dieta es adecuada.

Se observa un importante nivel de sedentarismo. Es conocida y está muy estudiada la asociación del sedentarismo con el aumento del $\mathrm{SM}^{27,28}$, y la gran mayoría de los participantes tienen una actividad limitada. El 81 '58\% pasan mucho tiempo sentados en una silla y delante de un ordenador, emplean su tiempo libre en ver la televisión o conectarse a Internet y casi la mitad duerme la siesta. Si salen a hacer ejercicio, optan sobre todo por el paseo. Incluso para acudir a trabajar, el 28 '95\% dice que lo hace andando, pero este hecho tiene aquí una peculiaridad y es que se trata de un acuartelamiento en el que hay viviendas dentro de su recinto, donde residen gran parte de los destinados en él, y prácticamente a todos los que acuden al trabajo andando les supone 300-400 m. de distancia, por lo que este dato no es valorable como actividad física. También hay que destacar que a medida que aumenta el riesgo de ECV, disminuye la intensidad y la cantidad de ejercicio que hacen.

Hay número importante de personas con sobrepeso y con un gasto energético insuficiente. Es importante procurar que estas personas consigan mantener un nivel adecuado de actividad física y una buena alimentación para conseguir un peso aceptable y así disminuir los factores de riesgo de ECV y un futuro más sano.

\section{CONCLUSIONES}

En el colectivo estudiado se ha podido observar que $7{ }^{\prime} 89 \%$ de los individuos tienen SM, todos varones; el 31'58\% tiene dos parámetros de diagnóstico del SM, es decir, son futuros candidatos a aumentar su riesgo de padecer ECV solo con que se modifique alguno de los parámetros que ahora tienen normales; más del $60 \%$ de los varones están por encima del normopeso; a mayor peso, mayor consumo de alimentos no saludables y, en general, mantienen una actividad más bien escasa, que 
además disminuye según aumentan los factores de riesgo cardiovascular. Todo esto nos sirve para poder decir que la falta de actividad física y los malos hábitos nutricionales actúan negativamente en el perfil lipídico, TA y el IMC del personal de la Guardia Civil que ha participado en el estudio, lo que aumenta la prevalencia del SM $\mathrm{y}$, por tanto, el riesgo de ECV.

Hay que tener en cuenta las limitaciones del estudio en cuanto a distribución de sexos, aunque este desequilibrio es inherente en este colectivo, pero los resultados nos sirven para plantearnos su repetición en otros acuartelamientos y para poner en marcha un plan de educación para la salud dirigido a que cambien sus hábitos en cuanto a ejercicio físico y hábitos alimentarios.

\section{REFERENCIAS}

1. Alimentación saludable y actividad física en la infancia y adolescencia.[Internet] Cantabria: Dirección General de Salud Pública. Gobierno de Cantabria; 2010. [Acceso: 30/08/20125. Disponible en:

http://www.saludcantabria.es/uploads/pdf/consejeria/Programa\%20Alimentacion.pdf

2. O.M.S. Obesidad y sobrepeso. [Internet] Nota descriptiva 311. Mayo 2012 [Actualizado 15/01/2015; Acceso 30/08/2015]. Disponible en:

http://www.who.int/mediacentre/factsheets/fs311/es/index.html\#

3. Yanett Palencia M. Alimentación y salud. Claves para una buena alimentación. [Internet][Acceso: 30/08/2015]. Disponible en:

http://www.unizar.es/med naturista/Alimentacion\%20y\%20Salud.pdf

4. Moral García J, Redecillas Peiro M, Martínez López E. Hábitos sedentarios de los adolescentes andaluces. J Sport Health Res. 2012;4(1):67-82.

5. Leal E, Aparicio D, Luti Y, Acosta L, Finol F, Rojas E, et al. Actividad física y enfermedad cardiovascular. Revista Latinoamericana de Hipertensión. 2009;4(1).

6. Ainsworth BE, HaskelL WL, Herrmann SD, Meckes N, Bassett DR, Tudor-Locke C, et al. 2011 Compendium of physical activities: a second update of codes and met values. Medicine and science in sports and exercise. 2011;43(8):1575-81.

7. OMS. Estrategia mundial sobre régimen alimentario, actividad física y salud ¿qué se entiende por actividad moderada y actividad vigorosa? who [internet]. 2011 [acceso: 30/08/2015]. disponible en:

http://www.who.int/dietphysicalactivity/physical activity intensity/es/index.html

8. Barrera Chuquiarque DE. Factores de riesgo para enfermedades cardiovasculares según los determinantes de la salud presentes en los choferes de transporte público 2014 [Tesis]. Lima-Perú: Universidad Nacional Mayor de San Marcos; 2015.

9. Gallego Gómez AM, Hita Contreras F, Lomas-Vega R, Martínez-Amat A. Estudio comparativo del índice de masa corporal y el equilibrio postural en estudiantes universitarios sanos. Fisioterapia [Internet]. 2011 [Acceso 31/08/2015]; 33(3):93-7. Disponible en: http://www.elsevier.es/es-revista-fisioterapia-146-articulo-estudiocomparativo-del-indice-masa-90024471.

10. Campillo Alameda FJ, López Ruiz P. La relación entre la obesidad y el ejercicio físico. EFDeportes.com [Internet]. 2010 [Acceso 29/08/2015]; 150. Disponible en:

http://www.efdeportes.com/efd150/la-relacion-entre-la-obesidad-y-el-ejercicio-

fisico.htm

11. Índice de masa corporal. MedlinePlus [Internet]. 2010. [Acceso: 31/08/2015]. Disponible en: http://www.nlm.nih.gov/medlineplus/spanish/ency/article/007196.htm

12. Sánchez-Contreras M, Moreno-Gómez G, Marín-Grisales M, García-Ortiz L. Factores de riesgo cardiovascular en poblaciones jóvenes. Rev Salud pública. 2009;11(1):2-11. 
13 Síndrome metabólico. Instituto del Corazón de Texas (Texas Heart Institute) [Internet]. 2012 [Actualizado 12/2014; Acceso 30/08/2015]. Disponible en: http://www.texasheartinstitute.org/HIC/Topics Esp/Cond/metabolic sp.cfm

14. Bello Rodríguez B, Sánchez Cruz G, Campos Ferreira Pinto A, Báez Pérez EG, Fernández Morín J, Achiong Estupiñán F. Síndrome metabólico: un problema de salud con múltiples definiciones. Rev Méd Electrón [internet]. 2012 mar-abr [acceso 29/08/2015]; 34(2). Disponible en: http://scielo.sld.cu/pdf/rme/v34n2/tema09.pdf

15. Cabezas G, Lares M, Velasco M, Rodríguez H, Albiarez I, Castro J, et al. Evaluación de marcadores antropométricos, bioquímicos y endoteliales de riesgo cardiovascular en individuos con síndrome metabólico, comparados con grupo control. síndrome cardiometabólico. 2012; II(1):P.6.

16. Fernández Ramos N. Prevalencia y caracterización bioquímica del síndrome metabólico en Canarias [tesis]. La Laguna: universidad de La Laguna; 2009.

17. Síndrome Metabólico. CardioSmart [Internet]. 2012 [Actualizado 22/05/2012; Acceso 30/08/2015]. Disponible en:

https://www.cardiosmart.org/healthwise/tm63/39sp/ec/tm6339spec

18. Villalobos Sánchez C, Mosquera Chacón J, Tovar Cortés H. Prevalencia del síndrome metabólico en consulta de medicina interna. Repert med CIR. 2011;20(2):93-102.

19. Cañón Barroso L, Díaz Herrera N, Calvo Hueros JI, Cruces Muro E, Nieto Hernández T, Buitrago Ramírez F. Incidencia de enfermedad cardiovascular y validez de las tablas de riesgo coronario en pacientes diabéticos con síndrome metabólico. Med Clin (Bar). 2007; 128(14):529-35.

20. Dapcich V, Salvador Castell G, Ribas Barba L, Pérez Rodrigo C, Aranceta Bartrina J, Serra Majem L. Guía de la alimentación saludable. Madrid: Sociedad Española de Nutrición Comunitaria; 2004.

21. López-González ÁA, Angullo Martínez E, Miguel Román Rodríguez M, VicenteHerrero MT, Tomás Salvá M, Ricci-Cabello I. Prevalencia de factores de riesgo cardiovascular en trabajadores aparentemente sanos. Gaceta Médica de México. 2012;148:430-7.

22. Gomis R, Artola S, Conthe P, Vidal J, Casamor R, Font B. Prevalencia en consultas de diabetes mellitus tipo 2 en pacientes con sobrepeso u obesidad en España. Estudio OBEDIA. Medicina clinica. 2013.

23. Pérez de Isla L, Saltijeral A, Vitale G, González B, Torres A, Álvarez-Sala L. Prevalencia de colesterol LDL inadecuado en pacientes con enfermedad coronaria y/o diabetes mellitus tipo 2. Rev Clin Esp. [Internet]. 2012 [Acceso 22/08/2015]; 212(10):475-81. Disponible en: http://www.revclinesp.es/es/prevalencia-colesterol-Idlinadecuado-pacientes/articulo/S0014256512003050/

24. Giacopini MI. LDL Pequeñas y Densas: Importancia de su Determinación. Tribuna del Investigador. 2010;11(1-2):20-6.

25. Ministerio de Sanidad, Servicios Sociales e Igualdad. Presentación de la encuesta nacional de salud: ense 2011-2012 [internet]; 2013. [acceso 30/08/2015]. disponible en:

http://www.msssi.gob.es/estadEstudios/estadisticas/encuestaNacional/encuestaNac20 11/PresentacionENSE2012.pdf

26. Ferrari MA. Metodología de investigación aplicada a encuestas nutricionales. Estimación de la ingesta de alimentos y/o nutrientes. Buenos Aires: Universidad de Buenos Aires, Escuela de Nutrición, Cátedra de Evaluación Nutricional; 2012. p. 3344.

27. Wijndaele K, Duvigneaud N, Matton L, Duquet W, Delecluse C, Thomis M, et al. Sedentary behaviour, physical activity and a continuous metabolic syndrome risk score in adults. European Journal of Clinical Nutrition. 2009;63:421-9. 
28. Edwardson CL, Gorely T, Davies MJ, Gray LJ, Khunti K, Wilmot EG, et al. Association of Sedentary Behaviour with Metabolic Syndrome: A Meta-Analysis. PLoS ONE. 2012;7(4): e34916.

Recibido: 2 de septiembre 2015; Aceptado: 2 de octubre 2015 